\section{Silencing factors participate in DNA repair and recombination in Saccharomyces cerevisiae}

\author{
Yasumasa Tsukamoto, Jun-ichi Kato \& Hideo Ikeda \\ Department of Molecular Biology, Institute of Medical Science, \\ University of Tokyo, P.O. Takanawa, Tokyo 108, Japan
}

DNA double-strand breaks are repaired by homologous recombination or DNA end-joining, but the latter process often causes illegitimate recombination and chromosome rearrangements. One of the factors involved in the end-joining process is Hdfl, a yeast homologue of Ku protein ${ }^{1-4}$. We used the yeast two-hybrid assay to show that Hdf1 interacts with Sir4, which is involved in transcriptional silencing at telomeres and $H M$ loci $^{5,6}$. Analyses of sir4 mutants showed that Sir4 is required for deletion by illegitimate recombination and DNA end-joining in the pathway involving Hdf1. Sir2 and Sir3, but not Sir1, were also found to participate in these processes. Furthermore, mutations of the SIR2, SIR3 and SIR4 genes conferred increased sensitivity to $\gamma$-radiation in a genetic background with a mutation of the RAD52 gene, which is essential for double-strand break repair mediated by homologous recombination. These results indicate that Sir proteins are involved in double-strand break repair mediated by end-joining. We propose that Sir proteins act with Hdf1 to alter broken DNA ends to create an inactivated chromatin structure that is essential for the rejoining of DNA ends.

Illegitimate recombination occurs between DNA sequences that are non-homologous or have very short regions of homology, and is the result of end-joining during the repair of DNA double-strand breaks. We have studied the formation of deletions resulting from illegitimate recombination in the yeast Saccharomyces cerevisiae, and found that the proteins Rad50, Mre11, Xrs2 and Hdf1 are involved in illegitimate recombination and end-joining ${ }^{1,2,7}$. Hdf1 is a yeast homologue of the $70 \mathrm{~K}$ subunit of the mammalian $\mathrm{Ku}$ protein, which can bind to the ends of double-stranded DNA and mediates double-strand break repair and immunoglobulin V(D)J recombination $^{8,9}$. The circularization of linear DNA by end-joining is less efficient in the yeast $h d f 1$ mutant and in cells with a mutation in the KU80 gene, which codes for a homologue of another subunit of the $\mathrm{Ku}$ protein ${ }^{3,4,10}$. The $h d f 1$ rad52 and $k u 80$ rad52 double mutants are more sensitive to $\gamma$-radiation than the isogenic rad52 single mutant, suggesting that most DNA double-strand breaks are repaired by end-joining in the absence of repair mediated by homologous recombination ${ }^{4,10,11}$. Mammalian $x r s 6$ mutant cells, which have a defect in one of the subunits of $\mathrm{Ku}$ protein, exhibit hypersensitivity to X-rays and are deficient in end-joining but not homologous recombination, indicating that end-joining is important for DNA double-strand break repair in mammalian cells ${ }^{12}$.

To investigate the function of Hdf1 in end-joining, we used the yeast two-hybrid system ${ }^{13}$ to search for yeast proteins that interact with Hdf1. Plasmid pLexHDF1, which carries the HDF1 gene fused to the DNA-binding-domain of LexA, was constructed and used for screening. From a library of genes fused to the Gal4 activation domain, 42 positive clones were isolated. Sequence analysis revealed that 11 of these clones had plasmids with the GAL4 fused in-frame to the SIR4 gene, which codes for a silencing information regulator. The Sir4 part of all fusions was found to begin at amino acid 1,205 of Sir4, and polymerase chain reaction (PCR) analysis confirmed that the sequence continued to the carboxy-terminal amino acid 1,358. The GAL4-SIR4 plasmid induced specific expression of the lac $Z$ reporter gene in the presence of the lexA-HDF1 plasmid (Table 1). Sir4 is a regulatory factor in transcriptional silencing at telomeres and $H M$ mating-type loci in yeast ${ }^{5,6}$. In addition to Sir4, the proteins Sir2, Sir3, Rap1 and histones $\mathrm{H} 3$ and $\mathrm{H} 4$ are necessary for silencing at telomeres ${ }^{5,6}$, and are thought to function by forming an inactivated chromatin structure analogous to the heterochromatin of higher eukaryotes ${ }^{5,6}$.

To study the role of Sir4 in illegitimate recombination, we first examined the effect of the sir4 mutation on deletion using a YCp plasmid, YCpL2 (ref. 7). YCpL2 carries two negative selection markers, the CAN1 and $C Y H 2$ genes, and three positive selection markers, the TRP1, LEU2 and URA3 genes. A can1 cyh2 strain containing YCpL2 exhibits sensitivity to both canavanine and cycloheximide, and deletion on the CAN1 and $\mathrm{CYH} 2$ genes on YCpL2 can be detected by selecting $\mathrm{Ura}^{+}$transformants resistant to both drugs. By structural analysis of the deleted plasmids, we have previously shown that deletion is caused by illegitimate recombination ${ }^{7}$. YCpL2 was introduced into a sir4 mutant and the rate of illegitimate recombination was determined. The rate was reduced 20-fold in the sir4 mutant compared to that of the isogenic SIR4 strain (Fig. 1a). We also tested the effects on deletion of

\begin{tabular}{|c|c|c|}
\hline $\begin{array}{l}\text { DNA-binding domain } \\
\text { plasmid }\end{array}$ & $\begin{array}{l}\text { Activation domain } \\
\text { plasmid }\end{array}$ & $\begin{array}{c}\beta \text {-Gal activity (s.d.) } \\
\left(\mathrm{nmol} \mathrm{min}^{-1} \mathrm{mg}^{-1} \text { protein }\right.\end{array}$ \\
\hline $\begin{array}{l}\text { pBTM116 } \\
\text { (LexA) } \\
\text { pBTM116 } \\
\text { (LexA) } \\
\text { pLexHDF1 } \\
\text { (LexA-Hdf1) } \\
\text { pLexHDF1 } \\
\text { (LexA-Hdf1) }\end{array}$ & $\begin{array}{c}\text { pGAD424 } \\
\text { (Gal4AD) } \\
\text { pGADSIR4 } \\
\text { (Gal4AD-Sir4) } \\
\text { pGAD424 } \\
\text { (Gal4AD) } \\
\text { pGADSIR4 } \\
\text { (Gal4AD-Sir4) }\end{array}$ & $\begin{array}{c}1.1(0.3) \\
1.7(0.4) \\
0.2(0.1) \\
20.9(3.8)\end{array}$ \\
\hline
\end{tabular}

The protein-protein interaction by two-hybrid assay was monitored by measuring the $\beta$ galactosidase activity of each $L 40$ transformants ${ }^{28}$. Results are an average of three determinations.
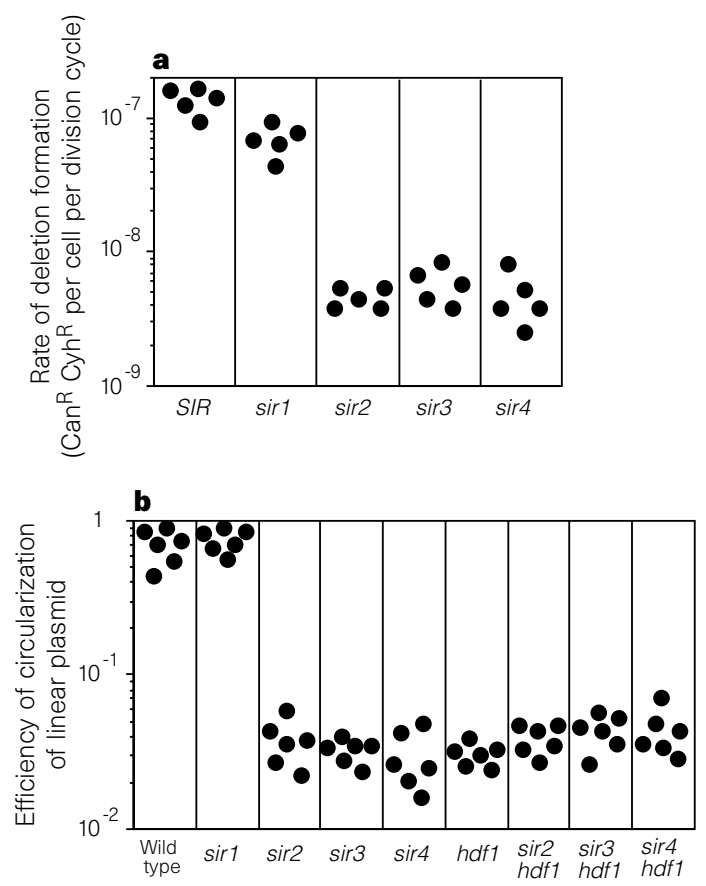

Figure 1 Deficiency in deletion formation and end-joining in sir mutants. a, Rate of deletion formation on the plasmid YCpL2. The recombination rate was determined by fluctuation analysis and data were analysed by the method of the median $^{29,30}$. b. The efficiency of end-joining of a linearized plasmid DNA. Endjoining efficiency was calculated on the basis of the ratio of pRS316 $(\mathrm{BamHI}$ digested; URA3) to YEp13 (uncut; LEU2) transformants. 
mutations in the SIR1, SIR2 and SIR3 genes. The rate of deletion formation was reduced in the sir 2 and sir 3 mutants, but not in the sir1 mutant, indicating that Sir2, Sir3 and Sir4, but not Sir1, are involved in illegitimate recombination.

To determine the function of Sir proteins in illegitimate recombination, we then used a dicentric plasmid to examine the effects of sir mutations on deletion. A dicentric plasmid is structurally unstable in yeast, and DNA double-strand breaks are thought to occur frequently on this plasmid as a result of the two resident centromeres being pulled to opposite poles during mitosis. It has been shown that deletion occurs at a high frequency, and requires functions implicated in end-joining ${ }^{2}$. Therefore, the frequency of deletions on the dicentric plasmid can be used as an indicator of the efficiency of end-joining. Because the dicentric plasmid YdCp2 has the CAN1 and CYH2 genes, deletions can be detected by the loss of these negative selection markers ${ }^{2}$. The sir1, sir2, sir3 and sir4 mutants were transformed by YdCp2 and the frequency of deletions on the YdCp2 plasmid was measured (Table 2). The frequency was reduced in the sir2, sir3 and sir4 mutants, as well as in the hdf1 mutant, both not in the sir1 mutant, indicating that Sir2, Sir3 and Sir4, but not Sir1, are involved in end-joining. Moreover, the frequency of deletions in the sir4 hdf1 double mutant was similar to that seen in the sir4 or hdf1 single mutant, suggesting that Sir4 and Hdf1 function in the same pathway during the joining of broken DNA ends.

a

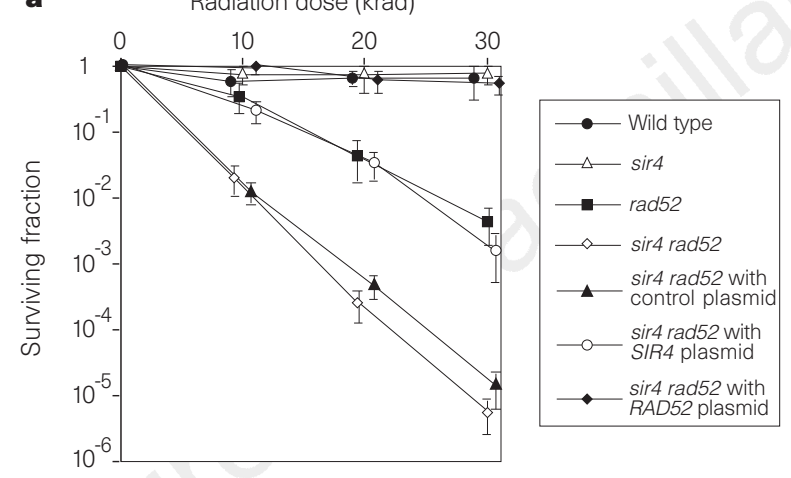

b

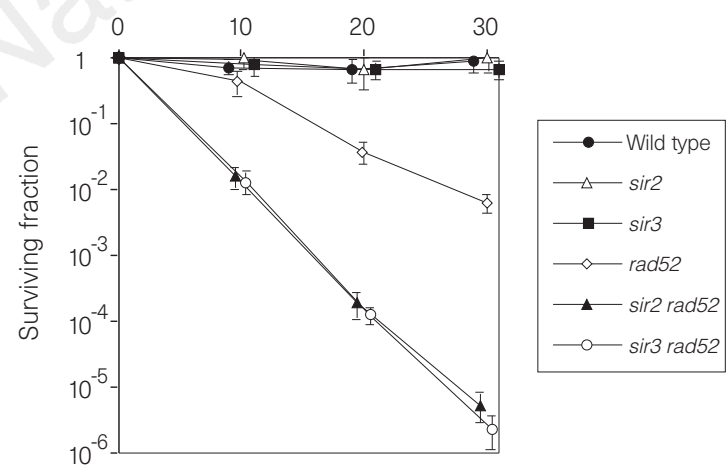

Figure 2 Sensitivity of sir and rad52 double mutants to $\gamma$-irradiation. a, Survival of the sir4 and rad52 single mutants and the sir4 rad52 double mutant after irradiation with $\gamma$-radiation. The control plasmid was the yeast vector pRS315 (ARSH4 CEN6 LEU2; ref. 14), and the SIR4 and RAD52 plasmids were the pRS315 containing the wild-type SIR4 and RAD52 genes, respectively. $\mathbf{b}$. Survival of the sir2, sir3 and rad52 single mutants and the sir2 rad52 and sir3 rad52 double mutants after $\gamma$-irradiation. W303-1A and isogenic derivatives were used in this experiment. Error bars represent one standard error. Results are an average of five independent determinations.
To confirm the involvement of Sir protein function in endjoining, we examined the efficiency of circularization of linear DNA fragments introduced into these sir mutants. BamHI digests of a URA3 plasmid, pRS316 (ref. 14), and an uncut LEU2 plasmid, YEp13 (ref. 15), were introduced into the ura3 leu2 strain. Because there is no homology, the linearized pRS316 plasmid cannot be recircularized by homologous recombination with chromosomal DNA, or with the uncut YEp13 plasmid. The efficiency of circularization was estimated on the basis of the ratio of the number of $\mathrm{Ura}^{+}$ colonies to that of Leu ${ }^{+}$colonies. In sir2, sir3, sir4 and $h d f 1$ mutants, the efficiency was reduced 20 -fold compared with the wild-type strain, whereas the efficiency was about the same in the sirl mutant (Fig. 1b). The deficiency of recircularization in the sir2, sir3 and sir4 mutants was complemented by the wild-type SIR2, SIR3 and SIR4 genes, respectively (data not shown). These results indicate that Sir2, Sir3 and Sir4 are essential for the rejoining of broken DNA ends, as shown previously for Hdf1. Because this result was consistent with the results of illegitimate recombination described above, the functions of Sir proteins involved in the end-joining step would be same as those for illegitimate recombination. The efficiency of circularization in the sir2 $h d f 1$, sir3 $h d f 1$ and sir4 $h d f 1$ double mutants was comparable to that in the sir2, sir3, sir4 or $h d f 1$ single mutants (Fig. 1b), confirming that Sir2, Sir3 and Sir4 work in the same pathway as Hdfl.

To investigate the effect of the sir4 mutation on the structure of end-joining products, the recombinant plasmids rescued from $\mathrm{Ura}^{+}$ colonies of the wild-type or the sir4 mutant were examined by restriction enzyme analysis. Of the 18 recombinant plasmids from the wild-type strain, 17 retained the BamHI site, whereas only 3 of the 17 recombinant plasmids derived from the sir4 mutant did so, indicating that most of the products from the sir4 mutant contain deletion mutations around the BamHI site. Analysis of the nucleotide sequence indicated that the recombinant plasmids derived from the sir4 mutant had deletions at various sites, and there were only short regions of homology (2-5 base pairs) at the recombination junctions (data not shown). These results indicate that the sir4 mutation reduces the frequency of precise end-joining in accordance with those with the $h d f 1$ and $k u 80$ mutations ${ }^{3,4,10}$. Consistent with a previous study ${ }^{4}$, the efficiency of circularization of the linearized pRS316 plasmids with blunt ends was about 10-fold lower than those with staggered ends in the wild-type strain (data not shown), indicating that DNA ends with short complementary sequences are effectively joined to each other in the Sir4-dependent end-joining pathway.

To investigate whether Sir proteins are involved in the repair of DNA double-strand breaks, we examined the sensitivity of the sir mutants to $\gamma$-radiation. Although the sir4 single mutant did not show increased sensitivity to ionizing radiation, the sir4 rad52 double mutant was more sensitive than the rad52 single mutant, and the radiation sensitivity of the sir4 rad52 double mutant was complemented by the plasmids containing the wild-type SIR4 or RAD52 genes (Fig. 2a). Similar results were obtained with the sir2 rad52 and sir3 rad52 double mutants (Fig. 2b). Because rad52 mutants are defective in DNA double-strand break repair mediated by homologous recombination ${ }^{16}$, these results indicate that the SIR2, SIR3 and SIR4 genes are involved in DNA double-strand break repair mediated by end-joining.

We have shown that Sir2, Sir3 and Sir4 are involved in illegitimate recombination, end-joining and DNA double-strand break repair, but that Sir1 is not. Sir2, Sir3 and Sir4 form a complex and are involved in silencing at telomeres and HM loci, whereas Sirl only makes a small contribution to silencing at HM loci and has no effect at telomeres ${ }^{5,6,17-19}$. Although the importance of silencing at telomeres is still not fully understood, silencing may protect telomeres from nuclease digestion by making telomeres inactive, as is the case for heterochromatin in higher eukaryotes ${ }^{5,6}$. DNA ends produced by double-strand breaks may also be protected by a mechanism similar 


\begin{tabular}{|c|c|c|c|c|}
\hline Genotype & $\begin{array}{c}\text { Transformation efficiency } \\
\text { Leu }^{+} \text {Ura }^{+} \text {per } \mu \mathrm{g} \\
\text { (YCpL2) }\end{array}$ & $\begin{array}{c}\text { Number of recombinants } \\
\text { Can }^{R} \text { Cyh }^{R} \text { Leu }^{+} \text {Ura }^{+} \text {per } \mu \mathrm{g} \\
\text { (YdCp2) }\end{array}$ & $\begin{array}{l}\text { Frequency of deletion } \\
\text { (s.d.) }\end{array}$ & Relative increase \\
\hline Wild type & $2.2 \times 10^{5}$ & 82 & $3.6(1.2) \times 10^{-4}$ & 1 \\
\hline sir1 & $3.2 \times 10^{5}$ & 90 & $3.6(0.8) \times 10^{-4}$ & 1.0 \\
\hline sir2 & $3.8 \times 10^{5}$ & 4.2 & $1.2(0.6) \times 10^{-5}$ & 0.033 \\
\hline sir3 & $1.1 \times 10^{5}$ & 1.2 & $8.6(6.0) \times 10^{-6}$ & 0.024 \\
\hline sir4 & $1.0 \times 10^{5}$ & 0.67 & $6.3(4.1) \times 10^{-6}$ & 0.018 \\
\hline$h d f 1$ & $3.4 \times 10^{5}$ & 2.8 & $8.3(6.7) \times 10^{-6}$ & 0.023 \\
\hline sir4 hdf1 & $1.2 \times 10^{5}$ & 0.83 & $6.6(4.0) \times 10^{-6}$ & 0.018 \\
\hline
\end{tabular}

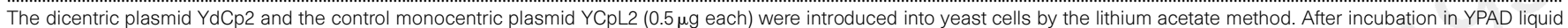

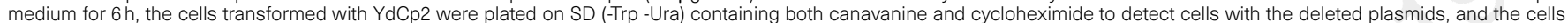
transformed with YCpL2 were plated on SD (-Trp -Ura) to measure transformation efficiency. Results are an average of six determinations.

to silencing at telomeres, and this process may be essential for endjoining. In telomere silencing, Sir2, Sir3 and Sir4 were found to lack DNA-binding activity, and did not seem to bind directly to DNA $^{5,6,20}$. The Rap1 protein, which binds to the specific sequences of telomeric regions, is thought to be required for them to bind to telomeres ${ }^{5,6,20}$. The results of the two-hybrid analysis suggested that Sir4 interacts with Hdf1. As the yeast Ku protein homologue can bind to the ends of double-stranded $\mathrm{DNA}^{8}$, Hdf1 may function by recruiting first Sir4, and then Sir2 and Sir3, which interact with Sir4, to the DNA ends produced by the double-strand break.

It is known that the sir3, sir4 and hdf1 mutants have shortened telomeres $^{21,22}$. Although a rap1 mutant has longer telomeres, Rap1 is also likely to be important in telomere maintenance by binding to telomeres $^{23,24}$. Because Rap1, Sir3 and Sir4 itself interact with the C terminus of Sir4, and we have shown that the C terminus of Sir4 interacts with Hdf1, we envisage that these proteins form a complex in telomeric regions of chromosomes and work together for telomere maintenance.

In addition to these Sir proteins and Hdf1, several other factors are essential for end-joining, including Rad50, Mre11 and Xrs2 (refs $2,7)$. However, the relation between the functions of these factors and those of Sir proteins is not yet understood. It is possible that the putative heterochromatin-like structure formed by the Hdf1 and Sir proteins might provide the prerequisite molecular context for the functioning of the Rad50-Mre11-Xrs2 complex in end-joining, but further work is required for the functions of Sir proteins in yeast end-joining to be understood. Our results also suggest that mammalian Sir-related proteins are involved in DNA double-strand break repair and recombination, such as the $\mathrm{V}(\mathrm{D}) \mathrm{J}$ recombination of the immunoglobulin gene. In mammals, transcriptional repression, such as $\mathrm{X}$-chromosome inactivation and genomic imprinting, share some similarities with silencing in yeast ${ }^{5}$. We expect that our understanding of the mechanism of end-joining will be also helped by studies into the functions of Sir protein homologues in mammals.

\section{Methods}

Strains. Recombination assays were performed in yeast strains DH6.61D (MATa trp1 his3 leu2 ura3 can1 cyh2; provided by J. W. Szostak) and its derivatives constructed by one-step gene replacement ${ }^{25}$ of the SIR1 gene with sir1::HIS3, the SIR2 gene with sir2::HIS3, the SIR3 gene with sir3 $\triangle::$ leu2::hisG, the SIR4 gene with sir4::TRP1, the HDF1 gene with hdf1 $\triangle::$ leu2:::his $G$ and the RAD52 with rad52A::hisG. Yeast strains W303-1A (MATa ade2-1 trp1-1 his3-11, 15 leu2-3, 112 ura3-1 can 1-100; ref. 26) and its derivatives were constructed as described above and were used to examine sensitivity to ionizing radiation.

Two-hybrid screening. The plasmid pLexHDF1 was constructed using the pBTM116 expression vector containing the Escherichia coli lexA gene under the control of the $A D H 1$ promoter and the TRP1 gene from the $2-\mu \mathrm{m}$ plasmid (provided by S. Fields). The plasmid generates a fusion protein of the LexA DNA binding domain joined to the full-length HDF1 gene amplified by PCR. To identify proteins interacting with Hdf1, we used three pools of a library of S. cerevisiae genomic DNA fragments fused to the Gal4 activation domain in pGAD plasmids ${ }^{13}$. Yeast strain L40 (MATa his3L200 trp1-901 leu2-3, 112 ade2 LYS2::(lexAop $)_{4}$-HIS3 URA3::(lexAop) $)_{8}$-lacZ; ref. 27) containing pLexHDF1 was transformed with the yeast library. After 3-4 days of growth on SC (-His-Trp-Leu) plates, $\mathrm{His}^{+} \mathrm{Trp}^{+} \mathrm{Leu}^{+}$transformants were replica-plated to SC (-His -Trp -Leu +X-gal) plates neutralized by potassium phosphate and incubated for 3 days until blue colonies appeared. Of the $7.9 \times 10^{6}$ transformants, 42 clones were isolated from the library.

Recombination assays. Determinations of the rate of illegitimate recombination on the plasmid YCPL2 and detection of plasmid deletion by end-joining using the dicentric plasmid YdCp2 were performed as described previously $y^{2,7}$. The transformation assay of linearized DNA was performed as described ${ }^{3}$. Briefly, a yeast strain was transformed by the lithium acetate method with a mixture of $300 \mathrm{ng}$ of linearized pRS316 DNA ${ }^{14}$ digested by BamHI and $100 \mathrm{ng}$ of uncut YEp13 DNA ${ }^{15}$ in the presence of $50 \mu \mathrm{g}$ of denatured salmon sperm DNA as carrier. The transformed cells were diluted and plated onto SC(-Ura) and SC(-Leu). Colonies were counted after incubation at $30^{\circ} \mathrm{C}$ for 3 days.

Measurements of sensitivity to ionizing radiation. Sensitivity of yeast strains to ionizing radiation was determined by exposing cell suspensions to a ${ }^{137}$ Cs source (Gammacell 40; Atomic energy of Canada) at $100 \mathrm{rad} \mathrm{min}^{-1}$. Irradiated and non-irradiated cells were diluted in water and spread on YPAD plates to monitor cell survival. Colonies were counted after plates had been incubated at $30^{\circ} \mathrm{C}$ for $2-4$ days. SIR 4 and RAD52 plasmids were constructed by cloning PCR-amplified fragments containing the SIR4 and RAD52 genes into SmaI site of the pRS315 plasmid ${ }^{14}$. The experiments were repeated five times.

Received 25 March; accepted 12 June 1997

1. Tsukamoto, Y., Kato, J. \& Ikeda, H. Hdf1, a yeast Ku-protein homologue, is involved in illegitimate recombination, but not in homologous recombination. Nucleic Acids Res. 24, 2067-2072 (1996).

2. Tsukamoto, Y., Kato, J. \& Ikeda, H. Budding yeast Rad50, Mre11, Xrs2, and Hdf1, but not Rad52, are involved in formation of deletion mutation on a dicentric plasmid. Mol. Gen. Genet. (in the press).

3. Milne, G. T., Jin, S., Shannon, K. B. \& Weaver, D. T. Mutations in two Ku homologs define a DNA endjoining repair pathway in Saccharomyces cerevisiae. Mol. Cell Biol. 16, 4189-4198 (1996).

4. Boulton, S. J. \& Jackson, S. P. Saccharomyces cerevisiae Ku70 potentiates illegitimate DNA doublestrand break repair and serves as a barrier to error-prone DNA repair pathways. EMBO J. 15, 50935103 (1996).

5. Laurenson, P. \& Rine, J. Silencers, silencing, and heritable transcriptional states. Microbiol. Rev. 56, $543-560$ (1992).

6. Shore, D. in Telomeres (eds Blackburn, E. H. \& Greider, C. W.) 139-191 (Cold Spring Harbor Laboratory Press, NY, 1995).

7. Tsukamoto, Y., Kato, J. \& Ikeda, H. Effects of mutations of RAD50, RAD51, RAD52, and related genes on illegitimate recombination in Saccharomyces cerevisiae. Genetics 142, 383-391 (1996).

8. Feldmann, H. \& Winnacker, E. L. A putative homologue of the human autoantigen Ku from Saccharomyces cerevisiae. J. Biol. Chem. 268, 12895-12900 (1993).

9. Weaver, D. T. What to do at an end: DNA double-strand-break repair. Trends Genet. 11, 388-392 (1995).

10. Boulton, S. J. \& Jackson, S. P. Identification of a Saccharomyces cerevisiae Ku80 homologue: roles in DNA double strand break rejoining and in telomeric maintenance. Nucleic Acids Res. 24, 4639-4648 (1996).

11. Siede, W., Friedl, A. A., Dianova, I., Eckardt-Schupp, F. \& Friedberg, E. C. The Saccharomyces cerevisiae $\mathrm{Ku}$ autoantigen homologue affects radiosensitivity only in the absence of homologous recombination. Genetics 142, 91-102 (1996).

12. Liang, F., Romanienko, P. J., Weaver, D. T., Jeggo, P. A. \& Jasin, M. Chromosomal double-strand break repair in Ku80-deficient cells. Proc. Natl Acad. Sci. USA 93, 8929-8933 (1996).

13. Chien, C. T., Bartel, P. L., Sternglanz, R. \& Fields, S. The two-hybrid system: a method to identify and clone genes for proteins that interact with a protein of interest. Proc. Natl Acad. Sci. USA 88, 95789582 (1991).

14. Sikorski, R. S. \& Hieter, P. A system of shuttle vectors and yeast host strains designed for efficient manipulation of DNA in Saccharomyces cerevisiae. Genetics 122, 19-27 (1989).

15. Broach, J. R., Strathern, J. N. \& Hicks, J. B. Transformation in yeast: development of a hybrid cloning vector and isolation of the CAN1 gene. Gene 8, 121-133 (1979).

16. Petes, T. D., Malone, R. E. \& Symington, L. S. in The molecular and Cellular Biology of the Yeast Saccharomyces. Genome Dynamics, Protein Synthesis, and Energetics Vol. 1 (eds Broach, J. R., Pringle, J. R. \& Jones, E. W.) 407-521 (Cold Spring Harbor Laboratory Press, NY, 1991).

17. Pilus, L. \& Rine, J. Epigenetic inheritance of transcriptional states in S. cerevisiae. Cell 59, 637-647 (1989). 
18. Aparicio, O. M., Billington, B. L. \& Gottschling, D. E. Modifiers of position effect are shared between telomeric and silent mating-type loci in S. cerevisiae. Cell 66, 1279-1287 (1991).

19. Moazed, D. \& Johnson, D. A deubiquitinating enzyme interacts with SIR4 and regulates silencing in S. cerevisiae. Cell 86, 667-677 (1996).

20. Moretti, P., Freeman, K., Coodly, L. \& Shore, D. Evidence that a complex of SIR proteins interacts with the silencer and telomere-binding protein RAP1. Genes Dev. 8, 2257-2269 (1994).

21. Palladino, F. et al. SIR3 and SIR4 proteins are required for the positioning and integrity of yeast telomeres. Cell 75, 543-555 (1993).

22. Porter, S. E., Greenwell, P. W., Ritchie, K. B. \& Petes, T. D. The DNA-binding protein Hdflp (a putative $\mathrm{Ku}$ homologue) is required for maintaining normal telomere length in Saccharomyces cerevisiae. Nucleic Acids Res. 24, 582-585 (1996).

23. Sussel, L. \& Shore, D. Separation of transcriptional activation and silencing functions of the RAP1encoded repressor/activator protein 1: isolation of viable mutants affecting both silencing and telomere length. Proc. Natl Acad. Sci. USA 88, 7749-7753 (1991).

24. Buck, S. W. \& Shore, D. Action of a RAP1 carboxy-terminal silencing domain reveals an underlying competition between HMR and telomeres in yeast. Genes Dev. 9, 370-384 (1995).

25. Rothstein, R. J. One-step gene disruption in yeast. Methods Enzymol. 101, 202-211 (1983).

26. Thomas, B. J. \& Rothstein, R. Elevated recombination rates in transcriptionally active DNA. Cell 56, 619-630 (1989)

27. Hollenberg, S. M., Sternglanz, R., Cheng, P. F. \& Weintraub, H. Identification of a new family of tissuespecific basic helix-loop-helix proteins with a two-hybrid system. Mol. Cell. Biol. 15, 3813-3822 (1995).

28. Rose, M. D., Winston, F. \& Hieter, P. Methods in Yeast Genetics, a Laboratory Course Manual (Cold Spring Harbor Laboratory Press, NY, 1990).

29. Luria, S. E. \& Delbruck, M. Mutations of bacteria from virus sensitivity to virus resistance. Genetics $\mathbf{2 8}$, 491-511 (1943)

30. Lea, D. E. \& Coulson, C. A. The distribution of the numbers of mutants in bacterial populations. J. Genet. 49, 264-285 (1948).

Acknowledgements. We thank S. Fields, S. M. Hollenberg, K. Johzuka, I. Kobayashi, A. Miyajima, J. Rine and J. W. Szostak for providing plasmids and strains, and K. Johzuka for advice on the two-hybrid assay. This work was supported in part by grants to Y.T., J.K. and H.I. from the Ministry of Education, Science, Sports, and Culture of Japan. Y.T. was supported by a postdoctoral fellowship of the Japan Society for the Promotion of Science.

Correspondence and requests for materials should be addressed to H.I. (e-mail: ike@hgc.ims.utokyo.ac.jp).

\section{Crystal structure of the} breakage-reunion domain of DNA gyrase

\author{
João H. Morais Cabral, Andrew P. Jackson, \\ Clare V. Smith, Nita Shikotra, Anthony Maxwell \\ \& Robert C. Liddington
}

Department of Biochemistry, University of Leicester, Leicester LE1 7RH, UK

DNA gyrase is a type II DNA topoisomerase from bacteria that introduces supercoils into $\mathrm{DNA}^{1,2}$. It catalyses the breakage of a DNA duplex (the $G$ segment), the passage of another segment (the $T$ segment) through the break, and then the reunification of the break. This activity involves the opening and closing of a series of molecular 'gates' which is coupled to ATP hydrolysis. Here we present the crystal structure of the 'breakage-reunion' domain of the gyrase at $2.8 \AA$ resolution. Comparison of the structure of this $59 \mathrm{~K}$ (relative molecular mass, 59,000) domain with that of a $92 \mathrm{~K}$ fragment of yeast topoisomerase II (ref. 3 ) reveals a very different quaternary organization, and we propose that the two structures represent two principal conformations that participate in the enzymatic pathway. The gyrase structure reveals a new dimer contact with a grooved concave surface for binding the $G$ segment and a cluster of conserved charged residues surrounding the active-site tyrosines. It also shows how breakage of the G segment can occur and, together with the topoisomerase II structure, suggests a pathway by which the $T$ segment can be released through the second gate of the enzyme. Mutations that confer resistance to the quinolone antibacterial agents cluster at the new dimer interface, indicating how these drugs might interact with the gyrase-DNA complex.

An alignment of domains in Escherichia coli gyrase and yeast topoisomerase II (topo II) is shown in Fig. 1, and their threedimensional structures are compared in Fig. 2. GyrA59 is the minimal fragment of the A-subunit which, when complexed with the B-subunit, has DNA-cleavage activity ${ }^{4}$. The carboxy-terminal domain of GyrA is required for the introduction of DNA supercoils, but the GyrA59-GyrB complex supports ATP-dependent relaxation of supercoils in a reaction reminiscent of conventional type II topoisomerases such as yeast topo II (ref. 5).

Each GyrA59 monomer is composed of an N-proximal head and a C-proximal tail. The head contains two domains: one similar to the DNA-binding domain of the catabolite-activator protein (CAP), including the helix-turn-helix (HTH) motif; and a second domain with $\alpha / \beta$ structure (the 'tower' domain) which stands on the CAP-like domain. The tail has a single domain with a helical core. Two long helices $(\alpha 14$ and $\alpha 18)$ emanate from this core and connect, together with the C-terminal helix $(\alpha 19)$, the head and tail fragments. The head fragment has a very similar structure to yeast topo II (ref. 3). The tail is also structurally conserved at its core, although large surface loops emanating from different points give it a different outward appearance. The tail fragments form the so-called 'primary' dimer interface (total buried surface, $2,150 \AA^{2}$ ), which is closely conserved in topo II (refs 3,6 ), providing a convenient reference point from which to compare the two structures.

The three connecting helices $(\alpha 14, \alpha 18$ and $\alpha 19)$ adopt very different conformations, leading to large quaternary movements involving a single hinge-point within the helices and rigid body movements of the head fragments (Fig. 2d). Compared with the yeast structure, the angle between $\alpha 18$ and $\alpha 19$ reduces from $120^{\circ}$ to $90^{\circ}$. At the same time, the long helix $\alpha 14$ bends by $30^{\circ}$ towards the dimer-related molecules and changes the way it packs against $\alpha 18$ and $\alpha 19$, shifting two helical turns on $\alpha 19$. The head fragments, which are rigidly connected to $\alpha 14$, swing towards each other and dock together to form a new contact (the 'head' dimer interface), with the active-site tyrosines moving by $26 \AA$ towards and past each other (Fig. 2a, b). The resultant structure is topologically a closed ring, with external dimensions of $100 \AA \times 40 \AA \times 100 \AA$ and a central hole of diameter $30 \AA$. The hole is much smaller than in the topo II structure but it is still large enough to accommodate a DNA duplex.

The 'head' dimer interface is dominated by an antiparallel sideby-side packing of the $\alpha 3$ helices (the first helix of the HTH motif) from each monomer, together with their adjoining loops (Fig. 3b, c). The helices pack very closely (mean centre-to-centre distance $7 \AA$ ) owing to a preponderance of small side chains. The two abutting surfaces are flat and hydrophobic at the centre, with charged groups at the periphery, burying a total surface area of $1,380 \AA^{2}$. At the top of the interface, the 'recognition' helices $(\alpha 4)$ make a head-to-head antiparallel dimer contact. Dimer formation creates a large concave surface $(100 \AA \times 30 \AA)$ on the top of the molecule formed by the upper surface of the CAP-like domains and the sides of the two 'tower' domains (Fig. 3a). The active-site tyrosines (Tyr 122) are on loops at either end of the dimer interface, $30 \AA$ apart (very similar to their separation in the yeast topo II structure), and sit at the ends of strongly basic grooves created by the dimer-related monomers. Previous biochemical studies have shown that the cleavage of DNA is achieved by a transesterification reaction between the tyrosines and the 'target' phosphoryl groups on opposing strands of the DNA backbone, resulting in the tyrosine being covalently attached to the $5^{\prime}$ end of the cleaved segment with a 4-base overhang ${ }^{1,2}$. The gyrase structure reveals a new cluster of conserved residues (Fig. 3c), juxtaposing Tyr 122 and Arg 121 from one monomer and His 80, Arg 32 and Lys 42 from the other monomer. We propose that this cluster forms the active site of the breakage-reunion reaction, with the other conserved positive charges (Arg 46 and Arg 47) anchoring the non-covalently bound $3^{\prime}$ end of the cleaved DNA.

We modelled a DNA duplex onto the GyrA59 structure by minimizing the distance between the tyrosines and the target phosphates, and by allowing the duplex to bend about a single 